\title{
Quantum field theory and experiments about vanishing of the zero-point vacuum energy
}

\begin{abstract}
Zahid Zakir *
Abstract

At quantization of relativistic fields the Hamiltonians symmetrized under the complex conjugate field operators lead to a zero-point vacuum energy. The standard recipe, introducing the operators of antiparticles, breaks the charge conjugation $(C)$ symmetry. For the Hamiltonians unsymmetrical under the field operators at exact $C$-symmetry requirement the vacuum does not contain a zeropoint energy and zero-point charge. The observable effects are described or as fluctuations of the physical vacuum with loop diagrams (Lamb shift), or as fluctuations of fields of real sources (Casimir effect). Both kind of vacuum fluctuations are created by the interaction Hamiltonians and they have no any relation to the zero-point fluctuations of (external) vacuum fields creating the zeropoint vacuum energy in the free Hamiltonians. Therefore, in fact the known experiments exclude the existence of the zero-point fluctuations of the pure vacuum fields and the zero-point vacuum energy and for the relativistic fields allow one to use only Hamiltonians unsymmetrized under the complex conjugate field operators with unbroken $C$-symmetry.
\end{abstract}

PACS: 03.70. + k; 11.10.-z; 11.30. Er

Key words: vacuum energy, vacuum fluctuations, Lamb shift, Casimir effect, time reversal, charge conjugation, cosmological constant

\section{Content}

Introduction

1 Charge-symmetric quantization of complex fields

1.1 A symmetry generating the zero-point energy of oscillator .........................................63

1.2 A symmetrized Hamiltonian of scalar field with the zero-point energy .........................64

1.3 Standard quantization with the unsymmetrized Hamiltonian: breaking of the charge conjugation symmetry

1.4 The unsymmetrized Hamiltonian without zero-point energy .........................................66

1.5 Quantization of spinor fields without a zero-point energy ............................................68

2 The experiments about lack of zero-point fluctuations of fields .............................................. 70

2.1 Three types of vacuum fluctuations........................................................................ 70

2.2 Lamb shift and Casimir effect exclude zero-point fluctuations of vacuum fields .............71

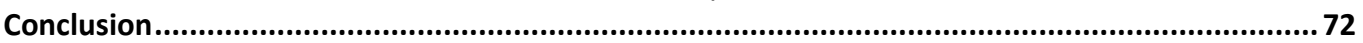

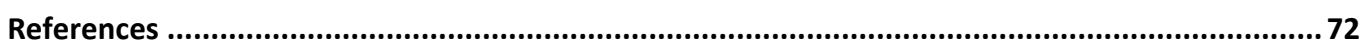

\section{Introduction}

In quantum field theory the zero-point vacuum energy plays a special role, being, probably, most contradictory of its predictions. As an illustration we present below the set of standard statements about that form of energy [1-3].

\footnotetext{
*Centre for Theoretical Physics and Astrophyics, Tashkent, Uzbekistan; zahidzakir@theor-phys.org
} 
Firstly, it has been believed that the zero-point vacuum energy is inevitable at the decomposition of fields on quantized harmonic oscillators.

But, since it is infinite, it has been removed from a free field Hamiltonian.

To justify such rough procedure, a shift of all energies to that value has been formally introduced by counting the energies from the zero-point vacuum energy. But in fact this justification does not work due to a gravitational effect of the such large energy, particularly, in cosmology.

Another justification of its removal was that this energy has no observable consequences. But this argument also turn out to be incorrect since the fluctuating vacuum fields, generating the zero-point energy, should be revealed in many observable effects.

In this connection then it has been announced, that the zero-point energy really is detected as the Lamb shift and Casimir effect and, moreover, that these effects are experimental proofs of the existence of the zero-point vacuum energy.

At the same time, these two effects are described exactly by other, real physical mechanisms, based on loop diagrams or fields of vibrating atoms [1-6].

In this connection then someone try to interpret that the description in terms of the zero-point fluctuations is only a heuristic description of the same physical phenomena with which the experiments can be explained without zero-point fluctuations of fields. But then there appears the question, where the zero-point fluctuations of pure vacuum fields as an independent physical phenomenon?

All these are only a part of attempts to find for the zero-point energy a place in the physical picture of the nature and although further they may be prolonged, inconsistencies and contradictions only increase $[3,6]$.

In the previous paper [7] it has been shown, that in fact for the relativistic fields the zero-point vacuum energy does not exist and all problems related by it have been created only by the historical reasons and unreasonable direct analogy with vibrations of a non-relativistic particle. It has been shown, that in the theory the introduction of the zero-point vacuum energy of relativistic fields does not necessary and, in fact, the experiments exclude it.

However, in [7] main results have not been clearly presented. In particular, in the presence of two types of mutually charge-conjugate quanta (particles and antiparticles) their states concern to two different subspaces of a total Fock space of a system. Therefore, the observables of quanta have a direct physical meaning only in their subspaces and one should be careful at summing them over the system. One must take into account that symmetric and unsymmetric combinations of canonical variables in the Hamiltonian differ to a value of the commutators in each subspace.

At a symmetry of the Hamiltonian under the canonically-conjugate variables the zero-point energy there is for both kind of quanta. Their contributions can be added only after reduction to the same sign of frequency and then the chargeconjugation symmetry becomes broken. If we choose a Hamiltonian without zeropoint energy for each kind of quanta, it appears as unsymmetrical under the canonical variables, while the charge conjugation symmetry becomes restored.

In the present paper the new method of charge-conjugation symmetric quantization of fields without zero-point energies will be described in the simplified, though quite rigorous, form. The confrontation with the experiments and main misunderstandings in the literature in the treatment of known experiments will be briefly discussed also. 


\section{Charge-symmetric quantization of complex fields}

\subsection{A symmetry generating the zero-point energy of oscillator}

The spectrum of a non-relativistic particle in the oscillatory potential contains a zero-point energy that is required by the uncertainty relations and experiments. In the formalism of the theory these physical requirements are satisfied by the symmetry of the Hamiltonian under the interchanging of canonically conjugate variables $p$ and $m \omega x$ :

$$
\begin{aligned}
H & =\frac{1}{2 m}\left[p^{2}+(m \omega x)^{2}\right]= \\
& =\frac{1}{4 m}[(m \omega x-i p)(m \omega x+i p)+(m \omega x+i p)(m \omega x-i p)] .
\end{aligned}
$$

Really, the canonical transformations to raising and lowering (ladder) operators $a, a^{*}$ preserve that symmetry and lead to a symmetrized product of ladder operators. Then, after a normal ordering of the operators in the symmetrized product, there appears the zero-point energy:

$$
H=\frac{1}{2} \omega\left(a^{*} a+a a^{*}\right)=\omega\left(a^{*} a+\frac{1}{2}\right) .
$$

However, as it is well-known, there exists also hermitian, but unsymmetrized product of these operators which is equivalent (1) at a classical level, but leads to another quantum Hamiltonian

$$
H_{(+)}=\frac{1}{2 m}(m \omega x-i p)(m \omega x+i p)=H+\frac{i}{2} \omega[x, p]=H-\frac{1}{2} \omega=\omega a^{*} a .
$$

As we see, this Hamiltonian does not contain a zero-point energy and, consequently, cannot be accepted as a Hamiltonian for a vibrating non-relativistic particle.

But, for oscillating systems, such as fields, which also can be represented as a set of quantum oscillators, it is possible to take or the symmetrized Hamiltonian $H$, if the experiments show that the system has a zero-point energy, or the unsymmetrized one $H_{(+)}$, if the zero-point energy had not observed. Therefore, the conventional statement that at a frequency decomposition of fields a zero-point energy of vacuum there appears inevitable is in fact an implicit postulation the existence of the such zero-point energy.

In fact, the theory of quantum oscillator supposes two kind of oscillations with and without the zero-point energy. The first mode is the case for the nonrelativistic particle, whereas for the quanta of relativistic fields with the chargeconjugation symmetry the existence of the zero-point energy should be studied additionally. The aim of the first part of the paper is to show that only a definite class of Hamiltonians of quantum fields contains the zero-point energy and that there exists also another class of Hamiltonians without a zero-point energy [7]. Therefore, only after the analysis of the experiments it will be possible to choose one of these classes of Hamiltonians and this question will be discussed in the second part of the paper. 


\subsection{A symmetrized Hamiltonian of scalar field with the zero-point energy}

In QFT a direct analogy with the spectrum of vibrating non-relativistic particle and the field modes exists only in the case of a symmetrized Hamiltonian containing the infinite zero-point energy. To the zero-point energy leads, particularly, the symmetrized Lagrangian for the charged scalar field:

$$
L_{s}=\frac{1}{2}\left[\left(\partial_{\mu} \varphi^{*}\right)\left(\partial^{\mu} \varphi\right)+\left(\partial_{\mu} \varphi\right)\left(\partial^{\mu} \varphi^{*}\right)-m^{2}\left(\varphi^{*} \varphi+\varphi \varphi^{*}\right)\right] .
$$

By decomposing the solutions of corresponding field equations on plane waves, it is necessary to take into account the positive and negative frequency states $k_{0}= \pm \omega$, where $\omega \equiv \omega_{k}=\left(\mathbf{k}^{2}+m^{2}\right)^{1 / 2}$ :

$$
\begin{aligned}
& \varphi(\mathrm{x})=\sum_{\mathbf{k}}\left[\tilde{a}(\omega, \mathbf{k}) e^{-i \omega t+i \mathbf{k x}}+\tilde{a}(-\omega,-\mathbf{k}) e^{i \omega t-i \mathbf{k x}}\right], \sum_{\mathbf{k}} \equiv \int \frac{\mathrm{d}^{3} k}{(2 \pi)^{3} 2 \omega}, \\
& \varphi^{*}(\mathrm{x})=\sum_{\mathbf{k}}\left[\tilde{a}^{*}(\omega, \mathbf{k}) e^{i \omega t-i \mathbf{k x}}+\tilde{a}^{*}(-\omega,-\mathbf{k}) e^{-i \omega t+i \mathbf{k x}}\right] .
\end{aligned}
$$

At the quantization, the field operators $\varphi(x), \varphi^{*}(x)$ and their time derivatives $\partial_{t} \varphi^{*}(x), \partial_{t} \varphi(\mathrm{x})$ obey the equal time commutation relations. From them we obtain the commutators for the creation-annihilation operators of field quanta, nonzero of which for the same frequency sign operators have the form:

$$
\begin{aligned}
& {\left[\tilde{a}(k), \tilde{a}^{*}\left(k^{\prime}\right)\right]=2 \omega(2 \pi)^{3} \delta^{3}\left(\mathbf{k}-\mathbf{k}^{\prime}\right),} \\
& {\left[\tilde{a}(-k), \tilde{a}^{*}\left(-k^{\prime}\right)\right]=-2 \omega(2 \pi)^{3} \delta^{3}\left(\mathbf{k}-\mathbf{k}^{\prime}\right),}
\end{aligned}
$$

The commutators of the operators with opposite frequency sign should vanish.

As the result, the symmetrized Hamiltonian, corresponding to the Lagrangian (4), in the momentum representation has the form

$$
H_{s}=\frac{1}{2} \sum_{\mathbf{k}} \omega\left[a^{*}(k) a(k)+a(k) a^{*}(k)+a^{*}(-k) a(-k)+a(-k) a^{*}(-k)\right] .
$$

The conventionally accepted postulate of QFT is the recipe of replacement of the operators $a(-k), a^{*}(-k)$ for the negative-frequency particles to the operators $b(k), b^{*}(k)$ for the positive-frequency antiparticles in the form:

$$
a(-k)=b^{*}(k), \quad a^{*}(-k)=b(k) .
$$

After the such replacement in the Hamiltonian there appears the zero-point energy $H_{(0)}$ :

$$
H_{s}=\frac{1}{2} \sum_{\mathbf{k}} \omega\left[a^{*} a+a a^{*}+b b^{*}+b^{*} b\right]=\sum_{\mathbf{k}} \omega\left(a^{*} a+b^{*} b\right)+H_{(0)} .
$$

Thus, here the zero-point energy arises due to the same reason, as in the case of vibrations of a non-relativistic particle, i.e. at the transition from the symmetrized products of the ladder operators to the normal products of these operators in both subspaces.

The problem of the negative norm of negative-frequency states is solved naturally as in the case of the harmonic oscillator (details see in [7]). 


\subsection{Standard quantization with the unsymmetrized Hamiltonian: breaking of the charge conjugation symmetry}

In QFT usually the standard Lagrangians, unsymmetrized under the replacement of field operators $\varphi^{*}, \varphi$, have been used also:

$$
L=\left(\partial_{\mu} \varphi^{*}\right)\left(\partial^{\mu} \varphi\right)-m^{2} \varphi^{*} \varphi .
$$

This Lagrangian has been considered intuitively as equivalent one to the Lagrangian (4), but it does not equivalent if we operate with eigenvalues of observable of particles and antiparticles only in their subspaces and at finding a relation between them one uses the charge conjugation symmetry requirements. The corresponding Hamiltonian and the charge operator have the form:

$$
\begin{aligned}
& H=\int \mathrm{d}^{3} x\left[\left(\partial_{t} \varphi^{*}\right)\left(\partial_{t} \varphi\right)+\left(\nabla \varphi^{*}\right)(\nabla \varphi)+m^{2} \varphi^{*} \varphi\right], \\
& Q=i \int \mathrm{d}^{3} x\left[\varphi^{*}\left(\partial_{t} \varphi\right)-\left(\partial_{t} \varphi^{*}\right) \varphi\right] .
\end{aligned}
$$

After the momentum decomposition of the fields, $H$ and $Q$ can be represented through the unsymmetrized products of the creation-annihilation operators:

$$
\begin{aligned}
& H=\sum_{\mathbf{k}} \omega\left[a^{*}(k) a(k)+a^{*}(-k) a(-k)\right], \\
& Q=\sum_{\mathbf{k}}\left[a^{*}(k) a(k)-a^{*}(-k) a(-k)\right] .
\end{aligned}
$$

In the standard approach there appeared the zero-point energy $H_{(0)}$ and the zero-point charge $-Q_{(0)}$ of vacuum as a result of the postulate on replacement of operators for negative-frequency particles to operators for positive-frequency antiparticles as in (8) by further normal ordering:

$$
\begin{gathered}
H=\sum_{\mathbf{k}} \omega\left[a^{*}(k) a(k)+b(k) b^{*}(k)\right]=\sum_{\mathbf{k}} \omega\left[a^{*}(k) a(k)+b^{*}(k) b(k)\right]+H_{(0)} \\
Q=\sum_{\mathbf{k}}\left[a^{*}(k) a(k)-b(k) b^{*}(k)\right]=\sum_{\mathbf{k}} \omega\left[a^{*}(k) a(k)-b^{*}(k) b(k)\right]-Q_{(0)}
\end{gathered}
$$

Unlike the symmetrized case, here the zero-point energy and the zero-point charge follow from the existence of negative-frequency modes of the field, or antiparticles, independently of the symmetries of the Lagrangian.

However, it can be shown that the such postulation of the direct replacement of operators according to (8) in expressions for $H$ and $Q$ leads to the breaking of the charge conjugation symmetry of the theory. Really, since the operators of antiparticles in our case are charge-conjugate to the operators of particles (up to inessential phase factors):

$$
C a(k) C^{-1}=b(k), \quad C a^{*}(k) C^{-1}=b^{*}(k),
$$

then, after the charge conjugation of the Hamiltonian $H$ and the charge $Q$, one obtains not their former expressions in the first lines of formulae (13) and (14), but new operators $H_{C}$ and $Q_{C}$ expressed through the operators of charge-conjugate particles: 


$$
\begin{aligned}
& H_{C} \equiv C H C^{-1}=\sum_{\mathbf{k}} \omega\left[b^{*}(k) b(k)+a(k) a^{*}(k)\right], \\
& Q_{C} \equiv C Q C^{-1}=\sum_{\mathbf{k}}\left[b^{*}(k) b(k)-a(k) a^{*}(k)\right] .
\end{aligned}
$$

Further we must take into account that the Hamiltonian of charge-symmetric fields does not vary at this transformation, while the charge operator should changes a sign:

$$
H=H_{C}, \quad Q=-Q_{C} .
$$

These conditions lead to the following relations between the operator products:

$$
\begin{aligned}
& a^{*}(k) a(k)+b(k) b^{*}(k)=b^{*}(k) b(k)+a(k) a^{*}(k), \\
& a^{*}(k) a(k)-b(k) b^{*}(k)=-b^{*}(k) b(k)+a(k) a^{*}(k) .
\end{aligned}
$$

By adding and subtracting these two equalities, we obtain strange relations:

$$
\begin{aligned}
& a^{*}(k) a(k)=a(k) a^{*}(k), \\
& b(k) b^{*}(k)=b^{*}(k) b(k) .
\end{aligned}
$$

Thus, the charge conjugation symmetry conditions would be obeyed only if the creation-annihilation operators commutate, which is impossible. This means, that the usual identification of operators according to (8) contradicts the charge conjugation invariance of the theory and, hence, it is inacceptable.

The breaking of the charge conjugation symmetry at the presence of the infinite zero-point charge of vacuum is not wonderful. Therefore, one of ways to restore the charge conjugation symmetry is to use the symmetrized Lagrangian (4), which leads to a vanishing zero-point charge of the vacuum. But, it leads to the divergent zero-point energy of the vacuum.

The standard way consists in a simply erasing of the zero-point energy in the Hamiltonian (13) and the zero-point charge in (14) by introducing a new postulate of normal ordering. However, the procedure of normal ordering does not solve the problem of breaking of the charge conjugation symmetry in initial expressions for the Hamiltonian and the charge operator, but it represents only a convention about ignoring the presence of this interior contradiction in the theory.

In the next section it will be shown, that these problems disappear in the case of unsymmetrized Lagrangians if we eliminate the products of the negative-frequency operators by means of some exact relations following from the charge conjugation symmetry properties of observables and that in this case the zero-point vacuum energy does not arise.

\subsection{The unsymmetrized Hamiltonian without zero-point energy}

The charge conjugation operation in (15) and also:

$$
C a(-k) C^{-1}=b(-k), \quad C a^{*}(-k) C^{-1}=b^{*}(-k),
$$

do not change a sign on frequency and relate only the same frequency-sign operators. Then, after the charge conjugation in (12), we obtain not the same operators $H$ and $Q$, but new operators $H_{C}$ and $Q_{C}$ expressed through the operators of charge-conjugate particles of both signs on frequency as: 


$$
\begin{aligned}
H_{C} & \equiv C H C^{-1}=\sum_{\mathbf{k}} \omega\left[b^{*}(k) b(k)+b^{*}(-k) b(-k)\right], \\
Q_{C} & \equiv C Q C^{-1}=\sum_{\mathbf{k}}\left[b^{*}(k) b(k)-b^{*}(-k) b(-k)\right] .
\end{aligned}
$$

Further, the charge conjugation symmetry conditions for the Hamiltonian and antisymmetry conditions for the charge operator (17) give us following relations between the operator products:

$$
\begin{aligned}
& a^{*}(k) a(k)+a^{*}(-k) a(-k)=b^{*}(k) b(k)+b^{*}(-k) b(-k), \\
& a^{*}(k) a(k)-a^{*}(-k) a(-k)=-b^{*}(k) b(k)+b^{*}(-k) b(-k) .
\end{aligned}
$$

By adding and subtracting these two equalities, we find the required identities between the products of boson operators:

$$
\begin{aligned}
& a^{*}(k) a(k)=b^{*}(-k) b(-k), \\
& a^{*}(-k) a(-k)=b^{*}(k) b(k) .
\end{aligned}
$$

Using these identities, we obtain final expressions for the Hamiltonian and the charge of the scalar field containing the contributions of positive-frequency particles and antiparticles only:

$$
\begin{aligned}
& H=\sum_{\mathbf{k}} \omega\left[a^{*}(k) a(k)+b^{*}(k) b(k)\right], \\
& Q=\sum_{\mathbf{k}}\left[a^{*}(k) a(k)-b^{*}(k) b(k)\right] .
\end{aligned}
$$

The similar procedure of the exclusion of negative-frequency operators in the case of the symmetrized Hamiltonian (7) leads to the symmetrized products of the positive-frequency operators (9) and there appear the positive zero-point energy for particles and antiparticles, although the zero-point charges cancel.

Thus, due to a correct taking into account the charge conjugation symmetry requirements, in the theory with unsymmetrized Lagrangian the operators of antiparticles appear automatically in a normal-ordered form and the zero-point energy and zero-point charge of vacuum do not arise.

Notice, that earlier in the standard QFT the same results have been obtained by the postulating of two compensating each other illegal operations:

(a) firstly, it has been postulated the direct replacement procedure (8) which then had led to the infinite zero-point vacuum energy and, in the case of unsymmetrized Lagrangians, to the zero-point charge breaking of the charge conjugation symmetry;

(b) secondly, it has been postulated the procedure of normal ordering of the operator products in observables, including the operation of erasing the divergent zero-point energy and the zero-point charge of vacuum.

In fact, in QFT a transition from products of operators for negative-frequency particles to products of operators for positive-frequency antiparticles can not be reduced to the linear replacements (8) because of non-linear character of the time reversal operation [7]. But, as it is shown above, in the case of free fields, for a correct exception of products of negative-frequency operators it is enough to use the charge conjugation symmetry conditions (17) for the observables without any new postulates. In the case of pseudoscalar fields, of course, it is necessary to use the similar to (17) CP-symmetry requirements. 


\subsection{Quantization of spinor fields without a zero-point energy}

Let's consider unsymmetrized (under the field operators) expressions for the Hamiltonian and the charge operator of spinor fields:

$$
\begin{aligned}
& H=\int d^{3} x\left[\frac{1}{2} \psi^{+} i \partial_{t} \psi-\frac{1}{2}\left(i \partial_{t} \psi^{+}\right) \psi+m \bar{\psi} \psi\right], \\
& Q=\int d^{3} x \psi^{+} \psi .
\end{aligned}
$$

The momentum decomposition of fields has the form:

$$
\begin{aligned}
& \psi(x)=2 m \sum_{\mathbf{k}, \alpha}\left[b_{\alpha}(k) u_{\alpha} e^{-i \omega t+i \mathbf{k x}}+b_{\alpha}(-k) v_{\alpha} e^{i \omega t-i \mathbf{k x}}\right], \\
& \psi^{+}(x)=2 m \sum_{\mathbf{k}, \alpha}\left[b_{\alpha}^{+}(k) u_{\alpha}^{+} e^{i \omega t-i \mathbf{k x}}+b_{\alpha}^{+}(-k) v_{\alpha}^{+} e^{-i \omega t+i \mathbf{k x}}\right] .
\end{aligned}
$$

At quantization, the creation-annihilation operators satisfy the anticommutation relations, non-zero of which are:

$$
\left\{b_{\alpha}( \pm k), b_{\alpha^{\prime}}^{+}\left( \pm k^{\prime}\right)\right\}=\frac{\omega}{m}(2 \pi)^{3} \delta^{3}\left(\mathbf{k}-\mathbf{k}^{\prime}\right) \delta_{\alpha \alpha^{\prime}},
$$

and, particularly, the different frequency sign operators anticommutate. After the substitution of momentum decomposition of fields, $H$ and $Q$ take the form:

$$
\begin{aligned}
& H=2 m \sum_{\mathbf{k}, \alpha} \omega\left[b_{\alpha}^{+}(k) b_{\alpha}(k)-b_{\alpha}^{+}(-k) b_{\alpha}(-k)\right], \\
& Q=2 m \sum_{\mathbf{k}, \alpha}\left[b_{\alpha}^{+}(k) b_{\alpha}(k)+b_{\alpha}^{+}(-k) b_{\alpha}(-k)\right] .
\end{aligned}
$$

If we accept the conventional postulate about a direct replacement of the negative-frequency operators in (27) as $b_{\alpha}(-k)=d_{\alpha}^{+}(k)$ and $b_{\alpha}^{+}(-k)=d_{\alpha}(k)$, then it easily can be shown that, as for the scalar field, in (29) there appear the divergent zero-point energy and the zero-point charge of vacuum and also the charge conjugation symmetry will be broken. Then we must to postulate the normal ordering of the operator products by erasing the divergences.

However, as for the scalar field, instead of these two compensating each other illegal postulates, we shall use simply the charge conjugation symmetry conditions for the observables. In terms of the corresponding charge-conjugate operators:

$$
\begin{array}{ll}
d_{\alpha}(k)=C b_{\alpha}(k) C^{-1}, & d_{\alpha}(-k)=C b_{\alpha}(-k) C^{-1}, \\
d_{\alpha}^{+}(k)=C b_{\alpha}^{+}(k) C^{-1}, & d_{\alpha}^{+}(-k)=C b_{\alpha}^{+}(-k) C^{-1},
\end{array}
$$

the charge-conjugate Hamiltonian $H_{C}$ and charge $Q_{C}$ take the form:

$$
\begin{aligned}
& H_{C}=2 m \sum_{\mathbf{k}, \alpha} \omega\left[d_{\alpha}^{+}(k) d_{\alpha}(k)-d_{\alpha}^{+}(-k) d_{\alpha}(-k)\right], \\
& Q_{C}=2 m \sum_{\mathbf{k}, \alpha}\left[d_{\alpha}^{+}(k) d_{\alpha}(k)+d_{\alpha}^{+}(-k) d_{\alpha}(-k)\right] .
\end{aligned}
$$

From them, using the charge conjugation symmetry of the Hamiltonian and antisymmetry of the charge (17), we obtain following relations for operator products: 


$$
\begin{aligned}
& b_{\alpha}^{+}(k) b_{\alpha}(k)-b_{\alpha}^{+}(-k) b_{\alpha}(-k)=d_{\alpha}^{+}(k) d_{\alpha}(k)-d_{\alpha}^{+}(-k) d_{\alpha}(-k), \\
& b_{\alpha}^{+}(k) b_{\alpha}(k)+b_{\alpha}^{+}(-k) b_{\alpha}(-k)=-d_{\alpha}^{+}(k) d_{\alpha}(k)-d_{\alpha}^{+}(-k) d_{\alpha}(-k) .
\end{aligned}
$$

By adding and subtracting these two equalities, we find the required operator identities for the fermionic operators:

$$
\begin{aligned}
& b_{\alpha}^{+}(k) b_{\alpha}(k)=-d_{\alpha}^{+}(-k) d_{\alpha}(-k), \\
& b_{\alpha}^{+}(-k) b_{\alpha}(-k)=-d_{\alpha}^{+}(k) d_{\alpha}(k) .
\end{aligned}
$$

As the result, the Hamiltonian and the charge operator for fermions can be expressed in terms of the positive-frequency operators as:

$$
\begin{aligned}
& H=2 m \sum_{\mathbf{k}} \omega\left[b_{\alpha}^{+}(k) b_{\alpha}(k)+d_{\alpha}^{+}(k) d_{\alpha}(k)\right], \\
& Q=2 m \sum_{\mathbf{k}}\left[b_{\alpha}^{+}(k) b_{\alpha}(k)-d_{\alpha}^{+}(k) d_{\alpha}(k)\right] .
\end{aligned}
$$

In the general case, including the chiral fields also, it is necessary to use the combined CP-symmetry conditions. The Hamiltonian and the charge operator of the chiral spinor fields, unsymmetrized under the field operators, are:

$$
\begin{aligned}
H=\int d^{3} x\left[\frac{1}{2} \psi_{L}^{+} i \partial_{t} \psi_{L}-\frac{1}{2}\left(i \partial_{t} \psi_{L}^{+}\right) \psi_{L}+\frac{1}{2} \psi_{R}^{+} i \partial_{t} \psi_{R}-\frac{1}{2}\left(i \partial_{t} \psi_{R}^{+}\right) \psi_{R}+\right. & \\
& \left.+m\left(\bar{\psi}_{L} \psi_{R}+\bar{\psi}_{R} \psi_{L}\right)\right],
\end{aligned}
$$

By using the $C P$-symmetry properties of the Hamiltonian and $C P$-antisymmetry of the charge:

$$
\begin{aligned}
& H_{C P} \equiv C P[H] P^{-1} C^{-1}=H, \\
& Q_{C P} \equiv C P[Q] P^{-1} C^{-1}=-Q .
\end{aligned}
$$

we obtain the relations between the operator products for each kind of chiral fields analogously to (33). As the result, one obtains $(h=L, R)$ :

$$
\begin{aligned}
& H=2 m \sum_{\mathbf{k}, h} \omega\left[b_{\alpha h}^{+}(k) b_{\alpha h}(k)+d_{\alpha h}^{+}(k) d_{\alpha h}(k)\right], \\
& Q=2 m \sum_{\mathbf{k}, h}\left[b_{\alpha h}^{+}(k) b_{\alpha h}(k)-d_{\alpha h}^{+}(k) d_{\alpha h}(k)\right] .
\end{aligned}
$$

Thus, at $C$ - or $C P$-symmetric quantization of the spinor fields with unsymmetrized Lagrangians the zero-point vacuum energy and the zero-point vacuum charge do not arise and the operators for observables here again appear automatically in the normal-ordered form.

In the former treatments a value of the vacuum expectation of the fermionic current operator had not vanish $[1,2]$ :

$$
\left\langle 0\left|j^{\mu}(x)\right| 0\right\rangle=\left\langle 0\left|\bar{\psi}(x) \gamma^{\mu} \psi(x)\right| 0\right\rangle \neq 0,
$$

and it was necessary to redefine the current by symmetrizing the field operators as $\left[\bar{\psi}, \gamma^{\mu} \psi\right]$, which is equivalent to the normal ordering $: \bar{\psi} \gamma^{\mu} \psi:$. But, then the free Lagrangian also should be symmetrized and this means that there appears the divergent zero-point vacuum energy. 
Let us consider the vacuum expectation value of the current in the new treatment without symmetrization and without zero-point energy. Substituting the momentum decompositions of fields into the expression for the current, we have:

$$
\begin{array}{r}
j^{\mu}(x)=(2 m)^{2} \sum_{k, k^{\prime}}\left[b_{\alpha}^{+}(k) b_{\alpha}\left(k^{\prime}\right) \bar{u} \gamma^{\mu} u^{\prime} e^{i\left(k-k^{\prime}\right) x}+b_{\alpha}^{+}(-k) b_{\alpha}\left(-k^{\prime}\right) \bar{v} \gamma^{\mu} v^{\prime} e^{-i\left(k-k^{\prime}\right) x}+\right. \\
\left.+b_{\alpha}^{+}(k) b_{\alpha}\left(-k^{\prime}\right) \bar{u} \gamma^{\mu} v^{\prime} e^{i\left(k+k^{\prime}\right) x}+b_{\alpha}^{+}(-k) b_{\alpha}\left(k^{\prime}\right) \bar{v} \gamma^{\mu} u^{\prime} e^{-i\left(k+k^{\prime}\right) x}\right] .
\end{array}
$$

The vacuum expectations from the first, third and fourth terms disappear automatically because of acting on the vacuum (at the left or on the right) of the positivefrequency operators. A remaining expectation from the second term with negativefrequency operators only also disappears due to the second of the operator identities (33):

$$
\begin{aligned}
& \left\langle 0\left|b_{\alpha}^{+}(-k) b_{\alpha}\left(-k^{\prime}\right)\right| 0\right\rangle \rightarrow\left\langle 0\left|b_{\alpha}^{+}(-k) b_{\alpha}(-k)\right| 0\right\rangle \delta^{3}\left(\mathbf{k}-\mathbf{k}^{\prime}\right)= \\
& =-\left\langle 0\left|d_{\alpha}^{+}(k) d_{\alpha}(k)\right| 0\right\rangle \delta^{3}\left(\mathbf{k}-\mathbf{k}^{\prime}\right)=0 .
\end{aligned}
$$

where the vanishing of anticommutators at different momentum is taken into account. Thus, we have:

$$
\left\langle 0\left|j^{\mu}(x)\right| 0\right\rangle=\left\langle 0\left|\bar{\psi}(x) \gamma^{\mu} \psi(x)\right| 0\right\rangle=0,
$$

i.e. in the theory with the unsymmetrized Hamiltonian the vacuum expectation value of the current operator vanishes without additional symmetrizations or postulating of the normal ordering.

\section{The experiments about lack of zero-point fluctuations of fields}

\subsection{Three types of vacuum fluctuations}

If, as in the conventional paradigm [3-4], we believe to the existence of zeropoint fluctuations of fields, then there appears a set of contradictions of this hypothesis with experiments. As it is well known, the Lamb shift and the Casimir effect can be explained completely as manifestation of zero-point fluctuations of vacuum fields. At the same time, these effects are described exactly as the results of interaction of particles or with virtual quanta of fields, or with fields of real sources without any reference to the zero-point fluctuations of vacuum fields.

The such peaceful co-existence of two explanations of the same phenomenon by two different physical reasons and not only on the same books, but in the mind of specialists, is wonderful by itself and requires more detailed discussion. Here it is necessary to distinguish, when given in the most of books and papers two types of explanations of these effects are the alternative methods of calculation of the same phenomena and when they are two independent mechanisms, the contributions of which to the observable effects are additive and should be added.

The first, with which we face at attempting to understand when who what means, is that the concept "vacuum fluctuations» in the literature has been used in various senses. Thus, usually one had started in view by one sense and then the obtained results had assigned to other sense of this concept. Moreover, the observation of the effects of one a type of vacuum fluctuations has been treated as a "proof" of the existence of in principle different type of fluctuations. Therefore, at the beginning we must define what types of vacuum fluctuations there exist.

At the first sense the notion "vacuum fluctuations" means the processes of creation and annihilation of the virtual quanta described by the loop diagrams. These 
fluctuations are the fluctuations of physical vacuum of quantized fields when field's quanta created in one a vertex of interaction and annihilate in another vertex. They are determined by the Hamiltonian of interaction $H_{I}$ at the vertexes of diagrams and the such loop fluctuations do not have any relation to the zero-point energy in the free Hamiltonian $H_{0}$.

The second sense of the notion "vacuum fluctuations» concerns the fluctuating external fields of real vibrating sources, particularly, atoms in solid states. These fluctuations are the fluctuations of fields of vibrating sources. Vibrating atoms in crystals can be presented as a set of harmonic oscillators (containing a zero-point energy) and electromagnetic fields of the such atoms, due to overlapping, form an effective fluctuating field of the crystal leading to many observable effects. Since they exist at zero temperature of the solid state, they also have been named as the zeropoint fluctuations. This fluctuating electromagnetic field of solid state again has not relation to the zero-point fluctuations of free electromagnetic field's vacuum, unrelated with any sources.

At last, at the third sense the concept "vacuum fluctuations» means the zeropoint fluctuations of vacuum fields generating the zero-point vacuum energy in the free Hamiltonian of fields $H_{0}$. Thus, it has been supposed, that if there is some energy of oscillations of fields, therefore, there are corresponding fluctuating pure vacuum fields, unrelated to any source and existing when there no field's quanta. Since these fluctuating fields do not relate with the creation or annihilation of quanta, they are some external fields. In the case of electromagnetic field the such fluctuating (external) vacuum fields $\mathbf{E}_{(0)}, \mathbf{H}_{(0)}$ have been introduced into the free Hamiltonian $H_{0}$ as [1-3]:

$$
\begin{aligned}
& H_{0}=\sum_{\mathbf{k}, \lambda} \omega a_{\lambda}^{*} a_{\lambda}+H_{(0)} \\
& H_{(0)}=2 V \int d^{3} k \frac{1}{2} \omega_{k}=\int_{V} d^{3} x \frac{1}{2}\left(\mathbf{E}_{(0)}^{2}+\mathbf{H}_{(0)}^{2}\right),
\end{aligned}
$$

where $V$ is a normalization volume. Notice again, that these fluctuations have no relation neither to quanta of fields, nor to sources, real or virtual and represent a new physical hypothesis in addition to the physical phenomena related by quanta or sources of fields.

The existence of the first two kinds of fluctuations of physical vacuum is obvious and their contributions to observable effects necessarily should be taken into account. Thus, the problem is, are their contributions enough for the description of observable effects and are exist the additional contributions of the third kind of fluctuations - truly zero-point fluctuations of pure vacuum fields.

\subsection{Lamb shift and Casimir effect exclude zero-point fluctuations of vacuum fields}

In the literature usually, the exact or the approximated calculations of the fluctuations of physical vacuum due to the loop diagrams (the Lamb shift and other effects of loop contributions), or effects of fields of real sources (the Casimir effect), have been considered. Thus, as the approximations of the exact contributions there have been introduced also some effective fluctuating fields, main contributions of 
which at certain model assumptions have a simple form. Thus, the results of the exact calculations of the contributions of the real sources are in excellent agreement with the experiments and the results of the approximating effective field also are close to the observable values.

However, historically the results of such approximate calculations of first two kind of experimentally confirmed fluctuations have been misappropriated to the third kind of fluctuations - to the zero-point fluctuations of the pure vacuum fields. Thus, misunderstanding also is related with a fact, that the simplifications of the real physical contributions appear as similar to contributions of qualitatively different physical phenomenon - the zero-point fluctuations of vacuum fields.

As the result, two illegal conclusions have been done: first, that the description of the effects in terms of the fluctuations of the physical vacuum (first two types of fluctuations) or in terms of the zero-point fluctuations of vacuum fields (the third type of fluctuations) have been treated as the different descriptions of the same phenomenon and secondly, that the observations of these effects confirm the existence of the zero-point fluctuations of vacuum fields.

The most known examples are two observable effects - the Lamb shift and the Casimir effect which are usually treated in the such dual form and it is widely accepted to refer them as to confirmations of the existence of the zero-point fluctuations of vacuum fields. In fact these effects are described exactly by the contributions of the first or second type of fluctuations [4-6] and there no room for the third one, to the hypothetical zero-point fluctuations of vacuum.

In other words, if we try to separate in each effect a pure contribution of the zero-point fluctuations of vacuum fields, which would be impossible to explain as an approximation of contributions of the loops or the fields of real sources, in each case the existence of such separate contribution has been rejected by the experiments [6].

\section{Conclusion}

Thus, only the Hamiltonians of relativistic fields with symmetrized under the complex conjugate field operators contain a divergent zero-point energy whereas the vacuum of the fields with unsymmetrized Hamiltonians does not contain the zeropoint energy and the zero-point charge due to the charge conjugation symmetry conditions.

The observable effects correspond or to the fluctuations of a physical vacuum related to the loop diagrams (the Lamb shift), or to the fluctuations of fields of real sources (the Casimir effect) and have no relation to in principle different physical phenomenon such as the zero-point fluctuations of vacuum fields. Therefore, the experiments exclude with high accuracy the existence of the zero-point vacuum energy of relativistic fields and allow us to use only the unsymmetrized Hamiltonians without the zero-point vacuum energy.

\section{References}

1. Bjorken J.D., Drell S.D. (1964) Relat. Quant. Mechanics, v.1, Mc Graw Hill,.

2. Itzykson C., Zuber J.-B. (1984) Quantum Field Theory, v.1, Mc Graw Hill.

3. Milonni P.W. (1994) Quantum Vacuum. Acad.Press.

4. Lifshitz E. M., Pitaevskii L. P. (1980) Statistical Physics (Pergamon), Part 2.

5. Barash Yu. S., Ginzburg V.L. (1975) Sov. Phys. Usp. 18, 305.

6. Jaffe R.L. (2005) Phys. Rev. D72, 021301; arXiv:hep-th/0503158.

7. Zakir Z. (2006) Theor. Phys., Astroph. \& Cosmol. 1, N 1, p.11; doi:10.9751/TPAC.2091-002 